\title{
Representative elementary volumes for evaluating effective seismic properties of heterogeneous poroelastic media
}

\author{
Marco Milani ${ }^{1}$, J. Germán Rubino², Tobias M. Müller ${ }^{3}$, Beatriz Quintal ${ }^{1}$, \\ Eva Caspari ${ }^{1}$, and Klaus Holliger ${ }^{1}$
}

\begin{abstract}
Understanding and quantifying seismic energy dissipation in fluid-saturated porous rocks is of considerable interest because it offers the perspective of extracting information with regard to the elastic and hydraulic rock properties. An important, if not dominant, attenuation mechanism prevailing in the seismic frequency band is wave-induced fluid pressure diffusion in response to the contrasts in elastic stiffness in the mesoscopic-scale range. An effective way to estimate seismic velocity dispersion and attenuation related to this phenomenon is through the application of numerical upscaling procedures to synthetic rock samples of interest. However, the estimated seismic properties are meaningful only if the underlying sample volume is at least of the size of a representative elementary volume (REV). In the
\end{abstract}

given context, the definition of an REV and the corresponding implications for the estimation of the effective seismic properties remain largely unexplored. To alleviate this problem, we have studied the characteristics of REVs for a set of idealized rock samples sharing high levels of velocity dispersion and attenuation. For periodically heterogeneous poroelastic media, the REV size was driven by boundary condition effects. Our results determined that boundary condition effects were absent for layered media and negligible in the presence of patchy saturation. Conversely, strong boundary condition effects arose in the presence of a periodic distribution of finite-length fractures, thus leading to large REV sizes. The results thus point to the importance of carefully determining the REV sizes of heterogeneous porous rocks for computing effective seismic properties, especially in the presence of strong dry frame stiffness contrasts.

\section{INTRODUCTION}

Ever since the pioneering work of Biot (1956a, 1956b), understanding and quantifying seismic attenuation in fluid-saturated porous rocks has continued to attract considerable interest. This ongoing interest is nourished by the possibility of extracting important hydraulic information from seismic data and certain properties of fracture networks (Pride 2005). One attenuation mechanism that is believed to play a defining role in the seismic frequency band is wave-induced fluid pressure diffusion, also referred to as wave-induced fluid flow (WIFF) (Pride et al., 2004; Mavko et al., 2009). This attenuation mechanism prevails when a seismic wave travels through a fluid-saturated porous medium containing heterogeneities in the mesoscopic scale range, i.e., heterogeneities larger than the typical pore sizes but smaller than the predominant seismic wavelengths. The passing seismic wavefield induces fluid pressure gradients in response to compliance contrasts associated with the heterogeneities. These fluid pressure gradients then equilibrate through pressure diffusion, and the viscous friction associated with the resulting relative fluid-solid motion causes energy dissipation, which in turn manifests itself in the form of velocity dispersion and attenuation in seismic recordings.

The numerical quantification of seismic properties in the presence of mesoscopic heterogeneities is not an easy task. This is mainly due to the fact that the predominant scales at which WIFF takes place, as characterized by the corresponding diffusion lengths, are much smaller than the scales at which wave propagation prevails. An effective means for exploring and quantifying the seismic

\footnotetext{
Manuscript received by the Editor 17 March 2015; revised manuscript received 25 September 2015; published online 01 March 2016.

${ }^{1}$ University of Lausanne, Applied and Environmental Geophysics Group, Institute of Earth Sciences, Lausanne, Switzerland. E-mail: marco.milani083@ gmail.com; beatriz.quintal@unil.ch; eva.caspari@unil.ch; klaus.holliger@unil.ch.

${ }^{2}$ Formerly University of Lausanne, Applied and Environmental Geophysics Group, Institute of Earth Sciences, Lausanne, Switzerland; presently University of Western Ontario, Department of Earth Sciences, London, Ontario, Canada. E-mail: jrubino9@uwo.ca.

Commonwealth Scientific and Industrial Research Organization, Perth, Australia. E-mail: tobias.mueller@csiro.au.

(C) 2016 Society of Exploration Geophysicists. All rights reserved.
} 
properties of such media is through the use of numerical upscaling procedures based on the theory of poroelasticity (Masson and Pride, 2007; Rubino et al., 2009). This is achieved by emulating for a subvolume of the heterogeneous porous material the action of a propagating seismic wavefield through oscillatory stress (the creep test) or displacement (the relaxation test) fields. Equivalent seismic properties in the form of phase velocity and attenuation can then be inferred from the spatially averaged stress and strain fields. A reasonable and important assumption upon which these upscaling approaches are commonly based is that the behavior of the probed heterogeneous poroelastic medium can be represented by a dynamically equivalent homogeneous viscoelastic medium (Solazzi et al., 2014; Jänicke et al., 2015). Because the size of the sample subjected to such upscaling procedures can be much smaller than the dominant seismic wavelength, these numerical strategies avoid the inherent problems related to the typically very large contrasts in spatial scale between the seismic wavelengths and the diffusion lengths. In the recent past, these upscaling approaches have allowed for exploring several new facets of WIFF, such as the effects of strong permeability fluctuations and fracture connectivity (Rubino et al., 2013a, 2013b, 2014), which are generally not amenable to analytical methods and are very difficult and costly to study through direct simulations of seismic wave propagation.

A key aspect of numerical upscaling procedures is that the resulting seismic properties are meaningful only if the size of the considered subvolume of the heterogeneous porous medium of interest is at least that of a corresponding representative elementary volume (REV). In the context of composite elastic solids, an REV is classically defined as a subvolume (1) that is structurally typical of

a)

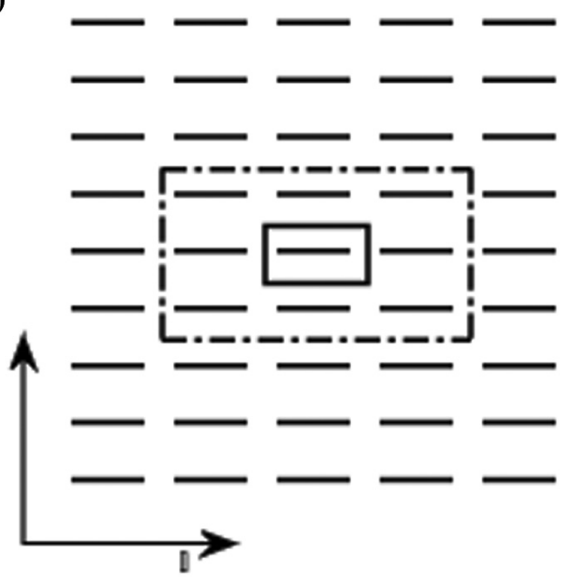

b)

c)
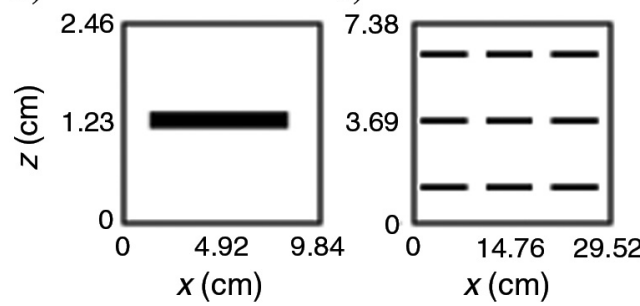

Figure 1. (a) Schematic representation of a periodically fractured medium and two examples of RUCs composed of (b) $1 \times 1$ and (c) $3 \times 3$ fundamental blocks. the whole medium on average and (2) for which the inferred properties are independent of the applied boundary conditions (Hill, 1963). The former condition essentially imposes statistical stationarity, whereas the latter implies that the seismic properties inferred from relaxation and creep tests must be equivalent. A key finding from corresponding works on elastic composites is that the rate of convergence of the seismic properties with respect to subvolumes of increasing sizes is expected to depend on the fracture density as well as on the compressibility contrast with respect to the background (Huet, 1990, 1999; Ostoja-Starzewski, 2006; Drago and Pindera, 2007; Gitman et al., 2007). Related studies on heterogeneous poroelastic media are as of yet largely unavailable. In one of the few works of this kind, Jänicke et al. (2015) investigate the effects of applying different boundary conditions to subvolumes of heterogeneous poroelastic media that were smaller than the corresponding REVs. In doing so, they mainly focus on the frequency shift of the attenuation peak caused by the different boundary conditions in the presence of WIFF at the boundaries. The rather small discrepancies observed between the P-wave phase velocities inferred from relaxation and creep tests are likely to be linked to the mild stiffness contrasts considered in their study.

The objective of this work is to explore boundary condition effects associated with the numerical upscaling of strongly heterogeneous porous media and the characteristics of the corresponding REVs. The answers to these questions are of significant practical importance because they form the basis for translating geologic subsurface scenarios of realistic complexity into equivalent viscoelastic seismic models. This in turn may open new avenues not only in seismic forward modeling, imaging, and AVO analysis but also with regard to processing techniques targeted toward strongly heterogeneous media, such as inverse $Q$ filtering. For the purpose of this pilot study, we consider periodic poroelastic media built by a sideby-side assembly of repeating unit cells (RUCs), which in turn are composed of fundamental blocks (Figure 1). The primary reasons for choosing periodic media in this pilot study are that (1) the first condition of Hill's (1963) definition of an REV is naturally satisfied for RUCs of any size and (2) such media tend to be particularly amenable for visualizing boundary condition effects and for analyzing their origins. We consider two fundamental scenarios typically associated with particularly strong WIFF effects: (1) porous media containing a periodic distribution of highly compliant fractures and (2) rocks saturated with patches of two types of pore fluids having differing compressibilities. We analyze the convergence of the Pwave phase velocity and attenuation obtained by applying relaxation and creep tests on RUCs composed of an increasing number of fundamental blocks. Because these two types of tests are expected to provide the lower and upper bounds of the apparent plane-wave modulus (Huet, 1990; Ostoja-Starzewski, 2006), the discrepancies observed between the resulting seismic properties enable us to analyze the convergence of the subvolumes to the corresponding REV. We also explore the sensitivity of boundary condition effects to changes in the fracture density and the compressibility contrasts between the fractures and the background.

\section{NUMERICAL UPSCALING METHODOLOGY}

\section{Equations of quasi-static poroelasticity}

The effects of WIFF in the mesoscopic scale range can be described by Biot's (1941) quasi-static poroelastic equations. The 
motivation for neglecting the inertial forces in Biot's (1956a, 1956b) dynamic equations is that, for frequencies much smaller than Biot's critical frequency, fluid flow is mainly controlled by fluid pressure diffusion. In the space-frequency domain, the corresponding system of two coupled equations, one describing the stress equilibrium within the sample, and the other describing Darcy-type flow, is given by:

$$
\begin{gathered}
\nabla \cdot \boldsymbol{\sigma}=\mathbf{0}, \\
i \omega \frac{\eta}{\kappa} \mathbf{w}=-\nabla p_{f},
\end{gathered}
$$

where $\eta$ is the fluid viscosity, $\kappa$ is the permeability, $\mathbf{w}$ is the relative fluid-solid displacement, and $\omega$ is the angular frequency. The total stress tensor $\boldsymbol{\sigma}$ of the material and the pore fluid pressure $p_{f}$ are given by (Biot, 1941)

$$
\begin{gathered}
\boldsymbol{\sigma}=\left(\lambda_{U} \nabla \cdot \mathbf{u}+\alpha M \nabla \cdot \mathbf{w}\right) \mathbf{I}+2 \mu \mathbf{\epsilon}, \\
p_{f}=-\alpha M\left(\nabla \cdot \mathbf{u}+\frac{1}{\alpha} \nabla \cdot \mathbf{w}\right),
\end{gathered}
$$

where $\mathbf{I}$ is the identity matrix, $\mathbf{\epsilon}=1 / 2\left(\nabla \mathbf{u}+(\nabla \mathbf{u})^{T}\right)$ is the strain tensor of the solid phase, and $\mathbf{u}$ denotes the solid displacement. The so-called Biot-Willis constant $\alpha$, the undrained Lamé parameter $\lambda_{U}$, and the fluid storage coefficient $M$ are given by

$$
\begin{gathered}
\alpha=1-\frac{K_{m}}{K_{s}}, \\
\lambda_{U}=K_{U}-\frac{2}{3} \mu, \\
M=\left(\frac{\phi}{K_{f}}+\frac{\alpha-\phi}{K_{s}}\right)^{-1},
\end{gathered}
$$

where $\phi$ is the porosity and $K_{f}, K_{s}$, and $K_{m}$ denote the bulk moduli of the fluid, the solid grains, and the dry frame, respectively. The undrained bulk and shear moduli $K_{U}$ and $\mu$ can be expressed as follows:

$$
\begin{gathered}
K_{U}=K_{m}+\alpha^{2} M, \\
\mu=\mu_{m},
\end{gathered}
$$

with $\mu_{m}$ denoting the dry frame shear modulus.

\section{P-wave phase velocity and attenuation inferred from relaxation and creep tests}

To estimate the $\mathrm{P}$-wave phase velocity and attenuation related to WIFF effects, we compute the stress and strain fields by solving equations 1-4 for boundary conditions corresponding to relaxation and creep tests. That is, we consider a $2 \mathrm{D}$ synthetic rock sample whose boundaries are hydraulically sealed. This implies that fluid is neither allowed to flow into nor out of the sample. The solid phase is neither allowed to have vertical displacements on the lower boundary nor horizontal displacements on the lateral ones. For the relaxation tests, a homogeneous time-harmonic vertical solid displacement

$$
u_{z}=-u^{0} e^{i \omega t}
$$

is imposed along the top boundary. For the creep tests, a global mean stress state is generated by imposing a homogeneous timeharmonic stress

$$
\sigma_{z z}=-\sigma^{0} e^{i \omega t}
$$

along the top boundary. A detailed description of the applied boundary conditions for the two tests is given in Appendix A.

Under the dynamic-equivalent viscoelastic medium assumption (Solazzi et al., 2014), the derivation of the equivalent viscoelastic properties of a heterogeneous poroelastic medium involves the spatial averaging of the inferred local stress and strain fields over the volume $V$ of the porous sample as follows:

$$
\begin{aligned}
\left\langle\sigma_{z z}(\omega)\right\rangle & =\frac{1}{V} \int_{V} \sigma_{z z}(\omega) d V, \\
\left\langle\epsilon_{z z}(\omega)\right\rangle & =\frac{1}{V} \int_{V} \epsilon_{z z}(\omega) d V .
\end{aligned}
$$

The corresponding equivalent undrained complex plane-wave modulus for the vertical direction of propagation is given by

$$
H(\omega)=\frac{\left\langle\sigma_{z z}(\omega)\right\rangle}{\left\langle\epsilon_{z z}(\omega)\right\rangle} .
$$

The equivalent $\mathrm{P}$-wave phase velocity $V_{\mathrm{P}}(\omega)$ and inverse quality factor $Q_{\mathrm{P}}^{-1}(\omega)$, which quantifies the attenuation, are then obtained as follows:

$$
\begin{gathered}
V_{\mathrm{P}}(\omega)=\left[\Re\left\{\sqrt{\frac{\langle\rho\rangle}{H(\omega)}}\right\}\right]^{-1}, \\
Q_{\mathrm{P}}^{-1}(\omega)=\frac{\mathfrak{s}\{H(\omega)\}}{\Re\{H(\omega)\}},
\end{gathered}
$$

where the average bulk density of the porous sample $\langle\rho\rangle$ is defined as follows:

$$
\langle\rho\rangle=\frac{1}{V} \int_{V}\left[\phi \rho_{f}+(1-\phi) \rho_{s}\right] d V,
$$

with $\rho_{f}$ and $\rho_{s}$ denoting the densities of the pore fluid and the solid grains, respectively.

\section{REV FOR PERIODICALLY HETEROGENEOUS POROELASTIC MEDIA}

\section{Fractured media}

We consider a poroelastic model corresponding to a watersaturated quartz sandstone containing periodically distributed mesoscopic fractures (Figure 1). We define a fundamental block containing one fracture. This corresponds to the RUC of minimum 
size and is denoted by the black rectangle (solid line) in Figure 1. Because of the symmetry of the considered model setup, this choice is consistent with undrained boundary conditions, which implies that no fluid flow occurs across the boundaries of the RUCs. In this scale range, it is reasonable to conceptualize fractures as porous media as opposed to void cavities because geologic observations suggest that natural fractures tend to be rough walled, with the walls being in contact at certain locations (Jaeger et al., 2007). In addition, the regions between the contact areas often contain rock fragments, weathering products, or mineral deposits. Therefore, in our simulations the fractures are modeled as rectangles of highly compliant material of very high porosity and permeability embedded in a stiff background of low porosity and permeability (Table 1). For the considered model setup, the value chosen for the permeability of the fractures does not play a significant role given that WIFF occurs between the fractures and the background (Rubino et al., 2014). The dimensions of the fundamental block and the embedded fracture are $9.84 \times 2.46$ and $4.84 \times 0.06 \mathrm{~cm}$, respectively, which correspond to a fracture volume fraction of approximately $1.2 \%$. The numerical domain is discretized with a regular mesh of square elements having a side length of $0.01 \mathrm{~cm}$. Following the methodology outlined above, we seek to determine the minimum number of fundamental blocks that are required to adequately approximate an REV. To do so, we compare the $\mathrm{P}$-wave phase velocities and inverse quality factors inferred from relaxation and creep tests applied to RUCs containing an increasing number of fundamental blocks.

\section{Phase velocity and attenuation}

Figure 2 shows the P-wave phase velocity and inverse quality factor for relaxation and creep tests applied to RUCs containing

Table 1. Physical properties of the fractures, the embedding background, and the saturating pore fluid. These parameters characterize a moderately tight fractured quartz sandstone, whose pore space is fully saturated by water. Adopted from Rubino et al. (2013a).

\section{Grain}

Bulk modulus, $K_{s}$

$37 \mathrm{GPa}$

Density, $\rho_{s}$

Background

Bulk modulus, $K_{m}^{b}$

Shear modulus, $\mu_{m}^{b}$

Porosity, $\phi^{b}$

Permeability, $\kappa^{b}$

Fracture

Bulk modulus, $K_{m}^{f}$

Shear modulus, $\mu_{m}^{f}$

Porosity, $\phi^{f}$

Permeability, $\kappa^{f}$

Water

Bulk modulus, $K_{w}$

Density, $\rho_{w}$

Viscosity, $\eta_{w}$
$1 \times 1,4 \times 4$, and $24 \times 24$ fundamental blocks. The dependence of the inferred seismic properties on the RUC size is evident in the results of creep tests because of the gradual convergence. Conversely, the rate of convergence of the results inferred from relaxation tests is so fast that they turn out to be largely independent of the RUC size. Indeed, a negligible maximum difference of approximately $0.1 \%$ is observed between the phase velocities inferred from relaxation tests applied to RUCs composed of $1 \times 1$ and $24 \times 24$ fundamental blocks.

Interestingly, for the phase velocity, the discrepancies between the results based on relaxation and creep tests are more pronounced in the low-frequency limit (approximately $0.1 \mathrm{~Hz}$ ) than in the highfrequency limit (approximately $1 \mathrm{MHz}$ ). In fact, a relative difference of approximately $23 \%$ is found between relaxation and creep tests for an RUC composed of $1 \times 1$ fundamental block in the low-frequency limit, whereas a relative difference of only approximately $2 \%$ is seen in the high-frequency limit. This is due to the fact that, in the low-frequency limit, there is enough time for the pore fluid to flow from the fractures into the host rock during the compression cycle, resulting in maximum deformation of the fractures. Conversely, in the high-frequency limit, there is not enough time for fluid exchange between the two regions, which results in a reduced deformation of the fractures. This stiffening effect correspondingly diminishes the compressibility contrast between the fractures and the background and thus causes a reduction of boundary condition effects. In the presence of significant WIFF, the discrepancies between relaxation and creep tests are therefore expected to be larger in the low frequencies as compared with the high-frequency limit,

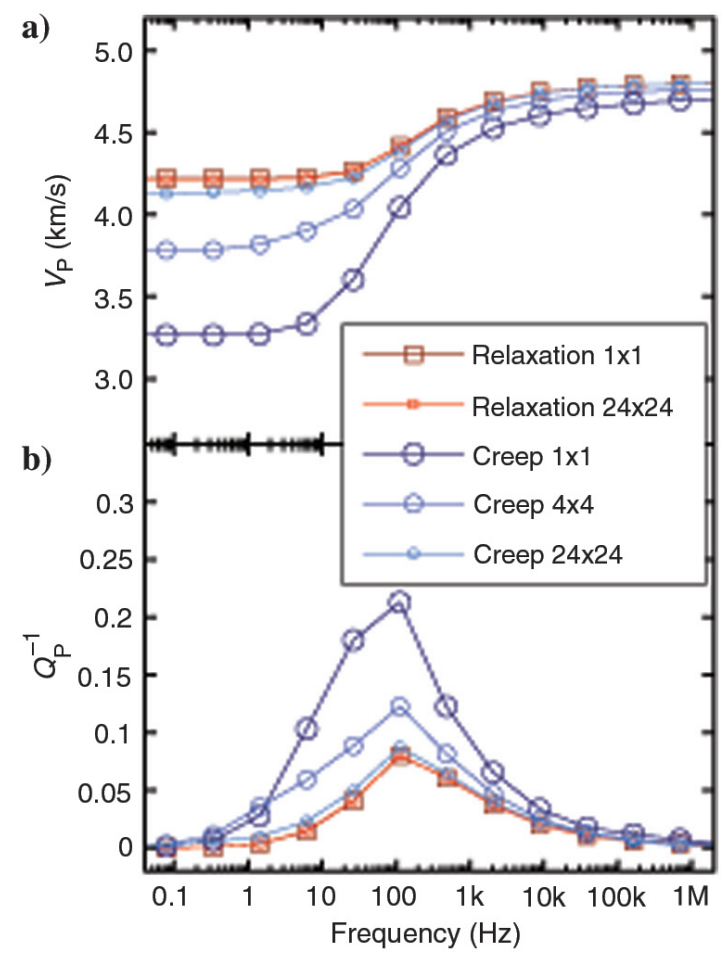

Figure 2. (a) P-wave phase velocity and (b) inverse quality factor as functions of frequency as inferred from relaxation (red) and creep (blue) tests for the case of periodically fractured media. The different symbol sizes relate to the number of fundamental blocks composing the considered RUC. 
where heterogeneous poroelastic media can be effectively replaced by corresponding heterogeneous elastic composites (Johnson, 2001). The decreasing discrepancies between relaxation and creep tests with frequency result in this case in a significant overestimation of the velocity dispersion inferred from creep tests (Figure 2a). As stipulated by the Kramers-Kronig causality relation, this overestimation of the velocity dispersion is associated with a corresponding overestimation of the attenuation for frequencies around its maximum. Figure $2 \mathrm{~b}$ shows that the amount of attenuation at the peak frequency obtained with a creep test for the RUC composed of $1 \times 1$ fundamental block is approximately 3.5 times higher than that obtained with a relaxation test. The frequency at which maximum attenuation occurs does not seem to be significantly affected by boundary condition effects. In this context, it is important to note that the frequency dependence of the differences between the phase velocities inferred from relaxation and creep tests and the associated overestimation of the attenuation inferred from creep tests are characteristics of poroelastic systems that have no equivalent in elastic composites.

Based on this convergence analysis, we see that an RUC composed of $24 \times 24$ fundamental blocks adequately approximates the size of an REV in a consistent manner with respect to Hill's (1963) definition. The fact that relaxation tests provide meaningful seismic properties already for an RUC composed of $1 \times 1$ fundamental block makes this test computationally less expensive than creep tests to obtain effective seismic properties. However, this extreme behavior is expected to be linked to the particular symmetry of the considered media and can a priori not be generalized for more complex heterogeneous media (Ostoja-Starzewski, 2006; Drago and Pindera, 2007).

\section{Stress and strain localizations}

In an attempt to understand the reasons for the discrepancies observed between relaxation and creep tests, we analyze the vertical components of the corresponding stress and strain fields. Due to the symmetry of the considered medium, these fields need to approach periodicity to make the inferred seismic properties independent from the applied boundary conditions (Drago and Pindera, 2007). We start focusing on the results in the low-frequency limit (approximately $0.1 \mathrm{~Hz}$ ), in which the largest differences in terms of phase velocity obtained from relaxation and creep tests occur (Figure 2). Figures 3 and 4 show the normalized stress and strain fields for two RUCs composed of $1 \times 1$ and $4 \times 4$ fundamental blocks as inferred from relaxation and creep tests, respectively. The used normalization factor is the average value of the corresponding field inferred from relaxation tests. For visualization purposes, and given that these fields are periodic in the horizontal direction, we only show the results for the fundamental blocks composing the first column of the corresponding RUC. In general, we notice that the presence of fractures results in highly heterogeneous stress and strain responses for both tests. In particular, we observe stress and strain amplification zones (red regions) around the fracture tips and shielding zones (blue regions) above and below the fractures. These patterns are characteristic of fractured elastic media (Knott, 1973; Grechka and Kachanov, 2006; Kundu, 2008). The considered geometry corresponds to the generic case of interacting fractures because the stress and strain features generated above and below (shielding zones) each fracture are influenced by and superimposed on those of the adjacent fractures (Figures 3 and 4).

Figure 3 shows that the application of a homogeneous displacement (relaxation test) at the top boundary of an RUC turns out to be compatible with an internal periodic rearrangement of stress and strain fields, regardless of the number of fundamental blocks. Conversely, the application of a homogeneous stress (creep test) at the top boundary directly interferes with the stress and strain fields. In particular, Figure $4 \mathrm{c}$ and $4 \mathrm{~d}$ shows that this boundary condition effect, which prevents a periodic distribution of the stress and strain fields, prevails in a small region beneath the top boundary. With increasing distance from this boundary, the fields resume periodicity and the effect of the disturbed region on the average stress and strain, and thus on the inferred seismic properties, eventually becomes negligible for a sufficiently large number of fundamental blocks.

Figure 5 shows the stress and strain fields inferred from relaxation (Figure 5a and 5b) and creep (Figure 5c and 5d) tests for an RUC composed of $4 \times 4$ fundamental blocks in the high-frequency limit. In this limit, the diminished compressibility contrast between the fractures and the background due to the stiffening effect of the pore fluid in undrained fractures significantly reduces the intensity of the stress and strain patterns associated with the fractures
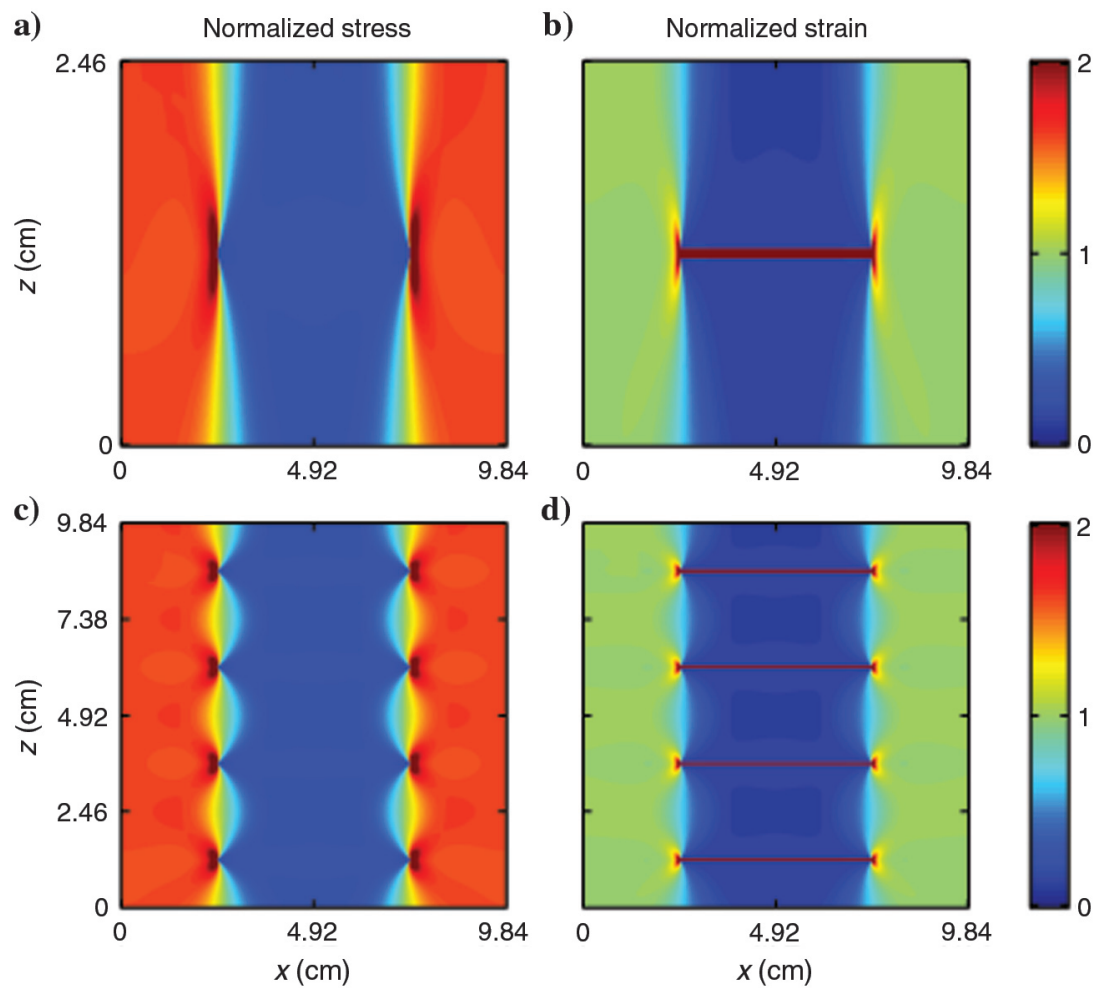

d)

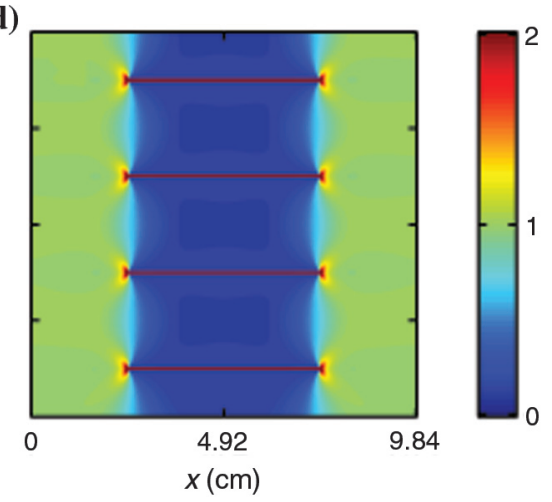

Figure 3. Normalized vertical (a and c) stress and (b and d) strain fields inferred from relaxation tests applied to RUCs composed of ( $a$ and b) $1 \times 1$ and (c and d) $4 \times 4$ fundamental blocks in the low-frequency limit for a periodically fractured medium. 
a)

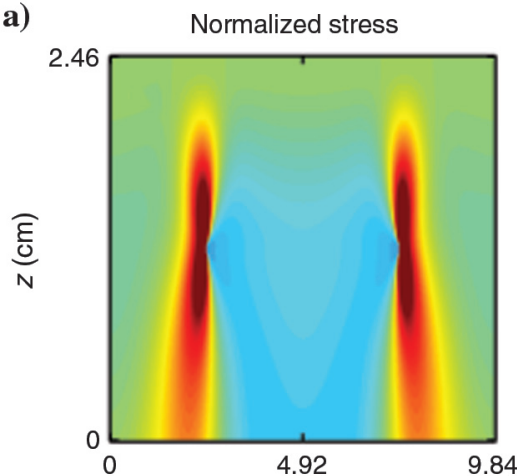

c)

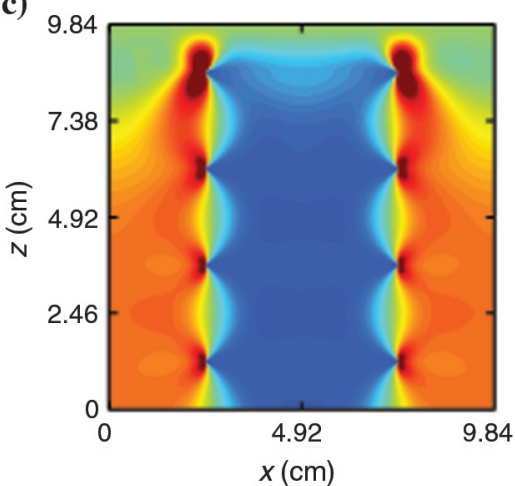

b)

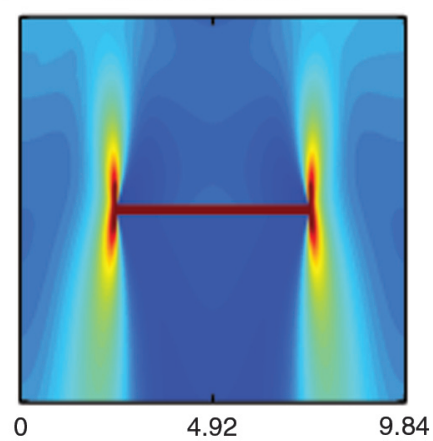

d)

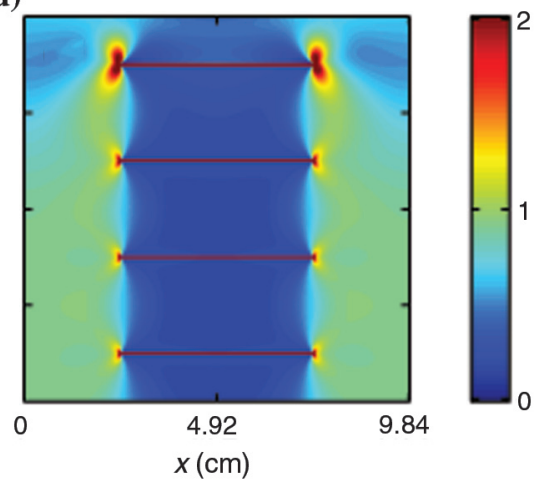

Figure 4. Normalized vertical (a and c) stress and (b and d) strain fields inferred from creep tests applied to RUCs composed of ( $a$ and b) $1 \times 1$ and ( $c$ and d) $4 \times 4$ fundamental blocks in the low-frequency limit for a periodically fractured medium.

a)

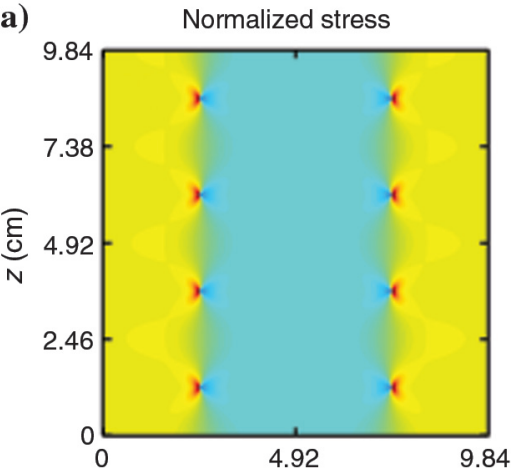

c)

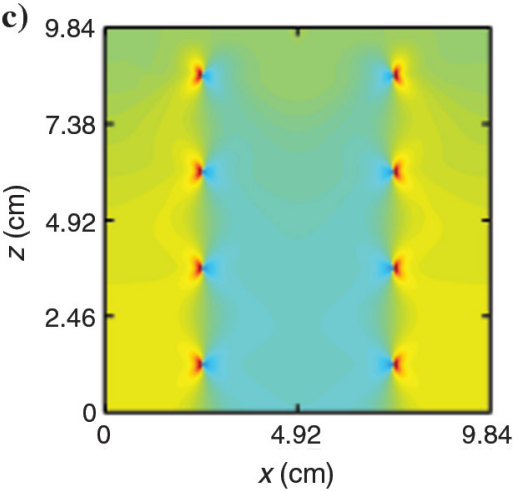

b)

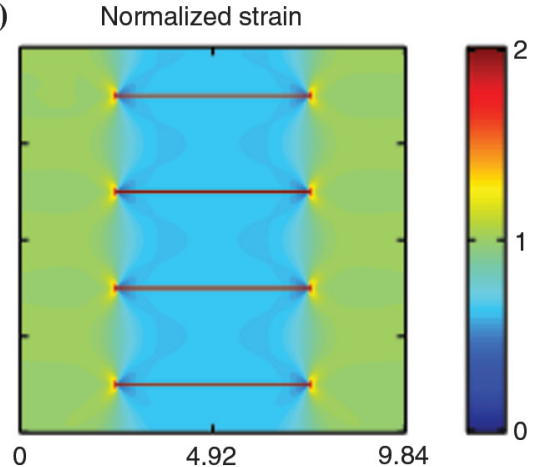

d)

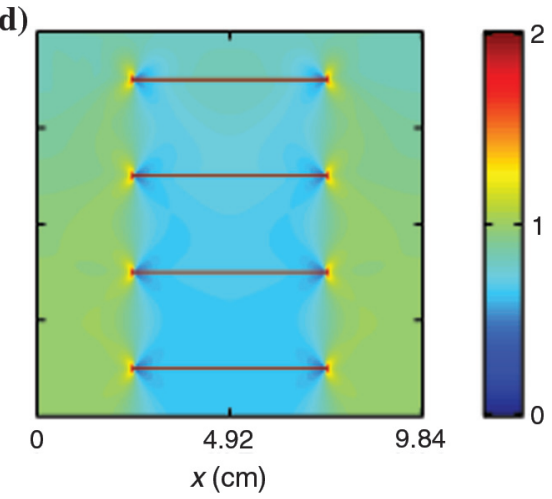

Figure 5. Normalized vertical (a and c) stress and (b and d) strain fields inferred from (a and b) relaxation and (c and d) creep tests applied to an RUC composed of $4 \times 4$ fundamental blocks in the high-frequency limit for a periodically fractured medium.

(Figure 5). As a consequence, the boundary condition effects occurring in creep tests are significantly reduced as well as the number of fundamental blocks required to average out this effect and to reach the REV size.

Another scenario for which boundary condition effects are mitigated, and thus the number of required fundamental blocks is reduced, is a medium with a lower fracture density. Here, we explore this situation by increasing the vertical side length of the fundamental block, which results in a larger distance between consecutive fractures. In Figure 6, we observe that the stress and strain features around the fractures are completely decoupled and no boundary condition effects occur. Indeed, in this limiting case of noninteracting fractures, one fundamental block is sufficient to represent the REV. The stress and strain patterns shown in Figures 3-6 therefore provide useful insights into the trends observed with regard to the inferred seismic properties as functions of the number of fundamental blocks.

\section{Sensitivity of the REV size to changes of the stiffness contrasts}

We now study the sensitivity of the REV size to changes of the dry frame stiffness contrasts between the fractures and the background. To do so, we change the dry frame elastic moduli of the fractures $K_{m}^{f}$ and $\mu_{m}^{f}$, whereas those of the background $K_{m}^{b}$ and $\mu_{m}^{b}$ remain unchanged. We assume $K_{m}^{f} / K_{m}^{b}=\mu_{m}^{f} / \mu_{m}^{b}$, and we consider ratios ranging between 1 and $10^{-4}$. The material and fluid properties are given in Table 1. Again, the dimensions of the fundamental block and the fracture are $9.84 \times 2.46$ and $4.84 \times 0.06 \mathrm{~cm}$, which corresponds to a fracture volume fraction of $1.2 \%$. We repeat the convergence analysis for the phase velocities obtained by performing relaxation and creep tests on RUCs composed of $1 \times 1,4 \times 4$, and $24 \times 24$ fundamental blocks.

Figure 7 shows the relative difference between the P-wave phase velocities inferred from relaxation and creep tests $\Delta V_{\mathrm{P}}=\left(V_{\mathrm{P}}^{\text {Relaxation }}-\right.$ $\left.V_{\mathrm{P}}^{\text {Creep }}\right) / V_{\mathrm{P}}^{\text {Relaxation }}$ as a function of the ratios between the dry frame elastic moduli of the fractures $K_{m}^{f}$ and $\mu_{m}^{f}$ and the background $K_{m}^{b}$ and $\mu_{m}^{b}$ for the low- and high-frequency limits. Based on these results, it is possible to assess whether the REV size is attained, which is the case when the discrepancy between relaxation and creep tests is negligible. For comparison, we include the case for a dry sample (black curve) composed of $1 \times 1$ fundamental block. Because there is no saturating fluids in the sample, WIFF does not take place and the result is frequency independent. Overall, we see that the REV size strongly depends on the dry frame stiffness contrasts. In particular, an RUC composed of $1 \times 1$ 
fundamental block adequately constitutes an REV for relatively mild contrasts $\left(K_{m}^{f} / K_{m}^{b}=\mu_{m}^{f} / \mu_{m}^{b} \geq 10^{-1}\right)$, whereas more fundamental blocks are required for higher contrasts. The reason for this is that, as we diminish the dry frame stiffness contrasts between the fractures and the background, the resulting heterogeneous stress and strain features around the fractures become less intense, the boundary condition effects are correspondingly less pronounced, and hence the number of fundamental blocks required for the corresponding REV size is also reduced. This behavior of the stress and strain fields with diminishing contrasts is very similar to the one for the transition from the low- to the high-frequency limit (Figures 3-5). It is interesting to observe that the increasing discrepancies between relaxation and creep tests as we increase the dry frame stiffness contrasts tend to asymptotic constant values. This is due to the fact that, as we reduce $K_{m}^{f}$ and $\mu_{m}^{f}$, the mechanical behavior of the mesoscopic fractures tends to that of void cavities.

We see that, the dry case represents the worst-case scenario in terms of boundary condition effects. The presence of a saturating fluid effectively reduces the stiffness contrasts between the fractures and the background, thus reducing boundary condition effects and the discrepancy between creep and relaxation test (compare black and blue curves). As expected, we also see that the discrepancies arising in the low-frequency limit are systematically higher than those in the high-frequency limit.

In the following section, we investigate whether boundary condition effects also occur for periodically layered media or in the presence of patchy saturation. The question regarding the definition of an REV also arises here because these scenarios are known to be capable of producing very significant velocity dispersion and attenuation due to WIFF in the mesoscopic scale range (Müller et al., 2010).

\section{Layered media}

To explore the characteristics of REVs in the presence of mesoscopic layering, we consider the case of a periodically layered system consisting of an alternating 1D water-saturated sequence of horizontal fractures and background material consisting of quartz sandstone (Table 1). The fractures have an aperture of $0.06 \mathrm{~cm}$ and are separated by $2.46 \mathrm{~cm}$ of background material, which corresponds to a fracture volume fraction of approximately $2.4 \%$. Figure 8 shows, for the lowfrequency limit, the normalized vertical stress and strain fields inferred from relaxation and creep tests applied to an RUC composed of $4 \times$ 4 fundamental blocks. We notice that, in this layered scenario, the stress fields are constant for both tests (Figure 8a and 8c), which can be explained by the fact that the total stress equilibrium (equation 1) reduces to

$$
\frac{\partial \sigma_{z z}}{\partial z}=0 .
$$

This in turn implies that the normal component of the total stress $\sigma_{z z}$ is constant within the samlimits. ple regardless of the applied boundary conditions and that lateral variations of the stress and strain fields cannot arise (Figure 8). As a consequence of the fact that each fundamental block composing the RUC is subjected to the same stress field, the generated strain field turns out to be periodic. This makes the two tests directly interchangeable, and therefore the seismic properties inferred from relaxation and creep tests considering RUCs composed of any number of fundamentals blocks are equivalent. The equivalence between the seismic properties inferred from relaxation and creep tests for an RUC composed of a $1 \times 1$ fundamental block is shown in Figure 9. Given that this finding is a direct consequence of the dimensionality of the system, it is universally valid for layered media, regardless of the material and fluid properties. This generic conclusion also holds in the presence of stochastic layering as long as Hill's (1963) first condition regarding statistical stationarity is satisfied.

\section{Patchy saturation}

Another pertinent scenario in the given context is patchy saturation, where the strong compressibility contrast between the saturat-
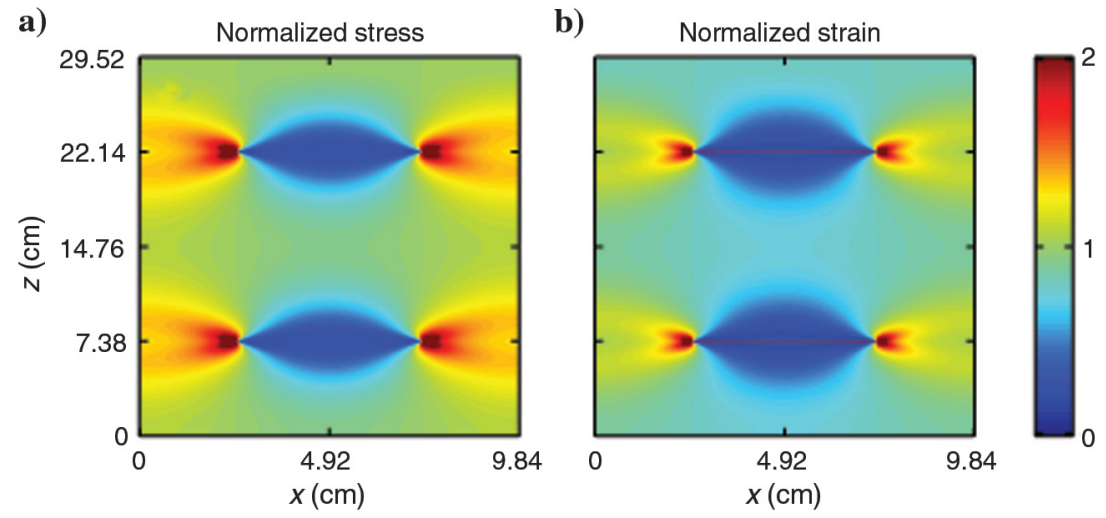

Figure 6. Normalized vertical (a) stress and (b) strain fields inferred from a creep test applied to an RUC composed of $2 \times 2$ fundamental blocks in the low-frequency limit for a periodically fractured medium.
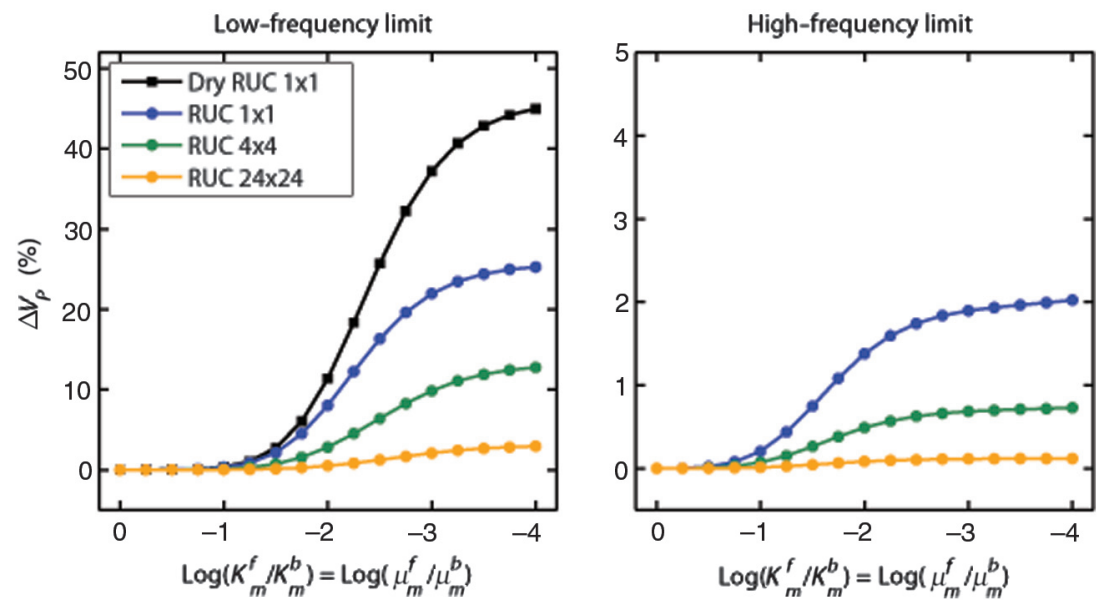

Figure 7. Relative difference between the P-wave phase velocities inferred from relaxation and creep tests as a function of the ratios between the dry frame elastic moduli of the fractures $K_{m}^{f}$ and $\mu_{m}^{f}$ and the background $K_{m}^{b}$ and $\mu_{m}^{b}$ in the low- and high-frequency 
a)

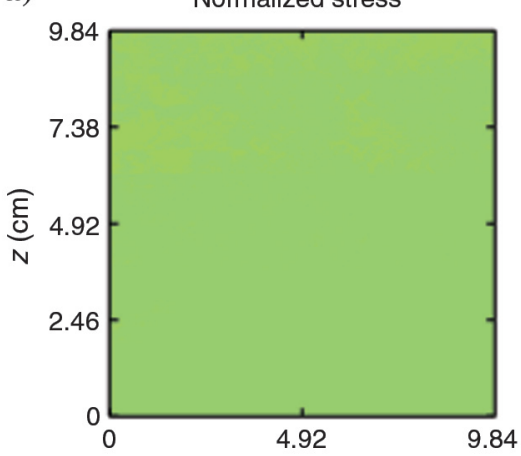

c)

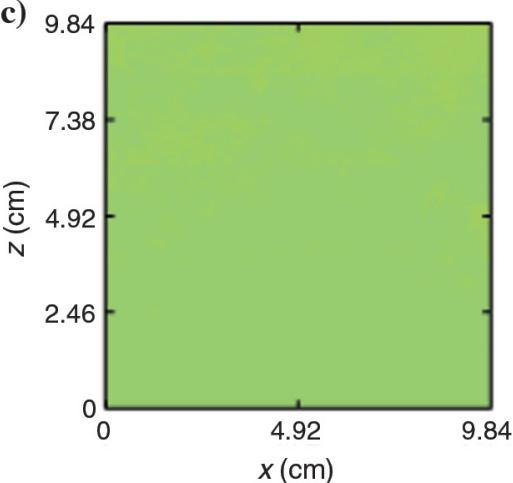

b)

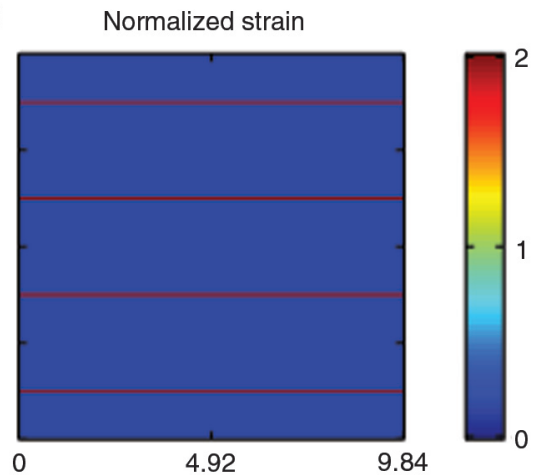

d)

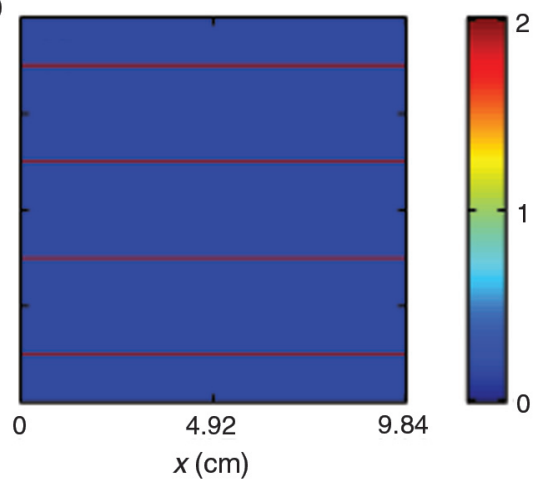

Figure 8. Normalized vertical (a and c) stress and (b and d) strain fields inferred from (a and b) relaxation and (c and d) creep tests in the low-frequency limit for a periodically layered medium.

a)

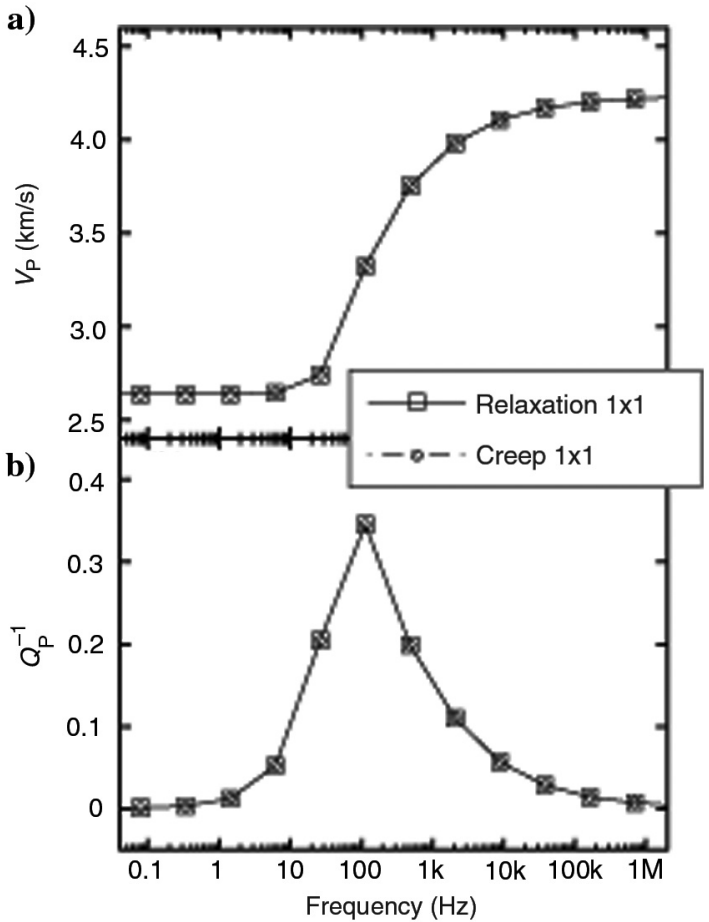

Figure 9. (a) P-wave phase velocity and (b) inverse quality factor as functions of frequency as inferred from relaxation (squares) and creep (circles) tests for a periodically layered medium. ing pore fluids can produce pronounced WIFF effects (White, 1975; Johnson, 2001). To explore the characteristics of REVs for these types of media, we define a simple fundamental block representing a $2 \mathrm{D}$ water-saturated sample with a circular gas patch in the center. In this case, we consider a homogeneous solid matrix. The dimensions of the fundamental block are $4.92 \times 4.92 \mathrm{~cm}$, and the overall gas saturation is $10 \%$, which is given by the ratio between the area of the gas-saturated patch and the total area of the fundamental block. Also in this case, the numerical domain is discretized with a regular mesh of square elements having a side length of $0.01 \mathrm{~cm}$. The fluid properties are given in Table 2. The material properties considered here correspond to those typical of an unconsolidated clean sand (Schön, 2004) with a porosity of 35\% (Table 2). Despite its simplicity, this model is highly diagnostic for assessing the importance of boundary condition effects in the presence of patchy saturation because it maximizes the corresponding WIFF effects.

In contrast to the results obtained for fractured media in the previous sections, we do not expect boundary condition effects in the low-frequency limit. The reason behind this is that, in this limit, the fluid pressure is equilibrated throughout the sample and the resulting stress and strain fields are expected to be homogeneous for a homogeneous solid matrix. Conversely, in the high-frequency limit, there is not enough time for pressure diffusion to occur between the central gas patch and the surrounding water-saturated region, which results in a piecewise constant pressure field (Johnson, 2001). This pressure pattern is associated with heterogeneous stress and strain fields that may interfere with the applied boundary conditions. In analogy with the results obtained for fractured media, we can argue that the heterogeneous stress and strain distributions arising around the gas patches tend to be more pronounced for higher stiffness contrasts between the water- and gas-saturated regions. In the high-frequency limit, these two regions can be represented as equivalent elastic solids having elastic bulk and shear moduli given by the undrained moduli of equations 8 and 9. It is then possible to quantify the stiffness contrasts between the water- and gas-saturated regions by quantifying the ratio between the corresponding undrained bulk moduli $K_{U}^{w} / K_{U}^{g}$ provided that the shear modulus is homogeneous, i.e., the ratio $\mu^{w} / \mu^{g}$ is equal to unity. Given that the ratio $K_{U}^{w} / K_{U}^{g}$ is much larger for unconsolidated materials than for consolidated rocks, it now becomes clear that our choice of an unconsolidated clean sand for the matrix was aimed at producing the highest conceivable stiffness contrasts between the water- and gas-saturated regions and thus the worst-case scenario.

Even though the extreme model setup considered here is expected to result in very heterogeneous stress and strain fields, Figure 10 illustrates the equivalence between the P-wave phase velocity and inverse quality factor inferred from relaxation and creep tests for an RUC composed of $1 \times 1$ fundamental block. This reflects the fact that boundary condition effects do not occur in this case as the resulting stress and strain fields turn out to be periodic for both tests 
(Figure 11a and 11b). The main reason for this is that there is only a contrast between the bulk modulus of the water and of the gas, whereas the shear modulus is homogeneous throughout the sample. Furthermore, we have verified that the effective P-wave modulus inferred from the numerical tests for the high-frequency limit coincides with that predicted by the analytical solution $H^{-1}=$ $\langle 1 / H\rangle$ (Hill, 1963; Johnson, 2001).

\section{Stress and strain localizations}

Figure 11a and $11 \mathrm{~b}$ shows heterogeneous stress and strain features around the gas patches for the high-frequency limit. Similar to fractured media, the strain field (Figure 11b) exhibits shielding (blue regions) and amplification (red regions) zones. Furthermore, we can observe that the strain patterns between adjacent gas patches interact with each other. In the stress field (Figure 11a), we can still identify weak amplification zones, whereas outside the gas patches, the stress field is rather homogeneous. This is different from what we observed for fractured media, and therefore, in the case of patchy saturation, no noticeable boundary condition effects arise for creep tests. As a consequence, applying either a homogeneous stress or a homogeneous displacement at the top boundary of the sample turns out to be compatible with a periodic rearrangement of the stress and strain fields, thus yielding the effective seismic properties for an RUC composed of $1 \times 1$ fundamental block.

For the sake of completeness, we repeated the present analysis considering the material properties corresponding to a typical sandstone. The results described above hold also for this case, and, as expected, Figure 11c and 11d demonstrates that the corresponding stress and strain fields are rather homogeneous. Another important result of this work is therefore that, as in the previously considered case of layered media, the definition of REV in the presence of patchy saturation can be solely based on Hill's (1963) first criterion of statistical stationarity. To further illustrate this, we have also verified the finding for a highly heterogeneous scenario characterized by a fractal-type distribution of the gas and water patches (Appendix B). The corresponding model and its results, which

Table 2. Physical properties of the homogeneous matrix and the two saturating pore fluids. These parameters characterize a clean sand partially saturated with water and gas.

\begin{tabular}{lc} 
Matrix & \\
Bulk modulus, $K_{m}^{b}$ & $0.37 \mathrm{GPa}$ \\
Shear modulus, $\mu_{m}^{b}$ & $0.44 \mathrm{GPa}$ \\
Porosity, $\phi^{b}$ & 0.35 \\
Permeability, $\kappa^{b}$ & $1 \mathrm{D}$ \\
Water & \\
Bulk modulus, $K_{w}$ & $2.25 \mathrm{GPa}$ \\
Density, $\rho_{w}$ & $1000 \mathrm{~kg} / \mathrm{m}^{3}$ \\
Viscosity, $\eta_{w}$ & $1.0 \times 10^{-3} \mathrm{~Pa} \cdot \mathrm{s}$ \\
Gas & \\
Bulk modulus, $K_{g}$ & $0.012 \mathrm{GPa}$ \\
Density, $\rho_{g}$ & $78 \mathrm{~kg} / \mathrm{m}^{3}$ \\
Viscosity, $\eta_{g}$ & $1.5 \times 10^{-4} \mathrm{~Pa} \cdot \mathrm{s}$ \\
\hline
\end{tabular}

are based on the material properties given in Table 2, are shown in Figure 12.

It is, however, important to notice that our findings are strictly valid only in the presence of a homogeneous matrix. The reason for this is that, in the given context, the combination of a heterogeneous solid matrix and patchy saturation is as of yet unexplored and hence boundary condition effects cannot be discarded for this scenario at present.

\section{DISCUSSION}

Understanding and exploring boundary condition effects related to the numerical upscaling of heterogeneous media through relaxation and creep tests and the implications for the definition of the corresponding REVs are of fundamental importance for the adequate interpretation of seismic velocity dispersion and attenuation measurements. The results shown in Figures 3-6 indicate that the mechanical interaction between consecutive fractures and, thus, the fracture density, is an important characteristic that control the strength of the boundary condition effects. In the case of interacting fractures, the stress and strain fields are characterized by lateral variations at the upper and lower boundaries of the fundamental blocks. This is incompatible with the homogeneous stress prescribed at the top boundary of the sample in creep tests and hence results in boundary condition effects. Conversely, in the case of noninteracting fractures, the stress and strain fields are rather homogeneous at the upper and lower boundaries of the fundamental blocks and hence are compatible with both relaxation and creep tests.

Boundary condition effects strongly depend on the stiffness contrast between the mesoscopic fractures and the background. This

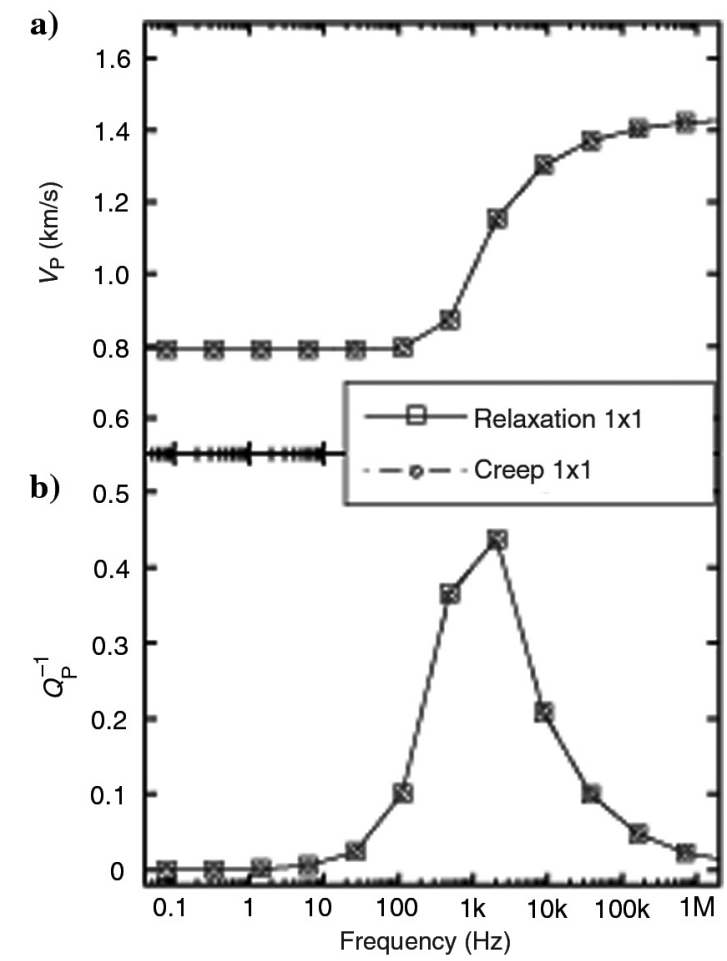

Figure 10. (a) P-wave phase velocity and (b) inverse quality factor as functions of frequency as inferred from relaxation (squares) and creep (circles) tests for the case of patchy saturation. 
contrast is determined by static poroelastic parameters, such as the presence of a saturating pore fluid and/or the difference between the dry frame elastic moduli of the background and the fracture. The worst-case scenario is therefore represented by dry samples in conjunction with fractures of very low dry frame elastic moduli (Figure 7). Saturating the sample with fluid implies a reduction of the boundary condition effects due to a reduction of the stiffness contrast between the fractures and the background. This stiffening effect of the fluid tends to dominate the stiffness of the saturated fractures as we reduce their dry frame elastic moduli.

Even though boundary condition effects strongly depend on the stiffness contrast, it is important to note that in the case of a homogeneous shear modulus no boundary effects occur. This has been verified by considering a sample with the fractures having the same dry frame shear modulus as the background; i.e., $\mu_{m}^{f}=\mu_{m}^{b}$. In this
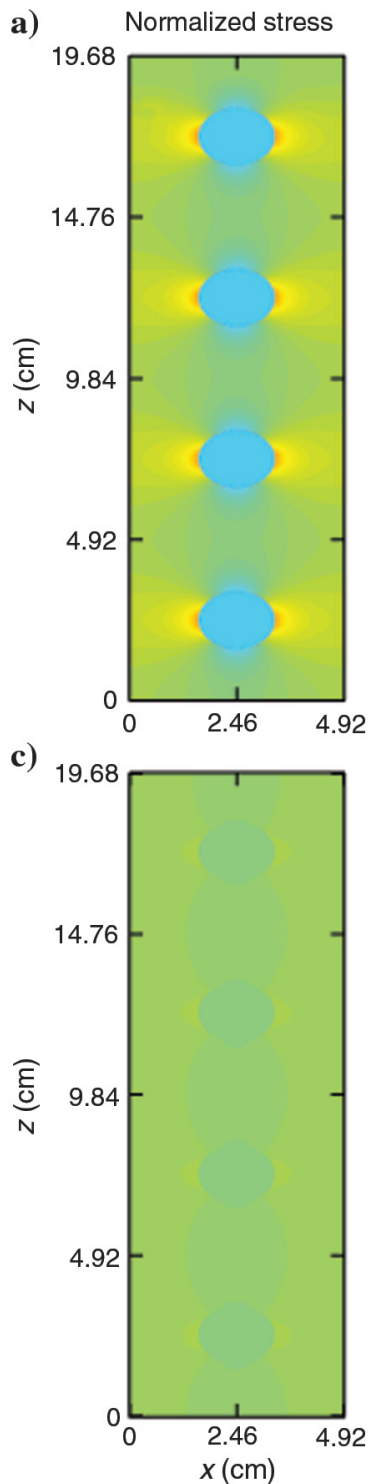

b) Normalized strain
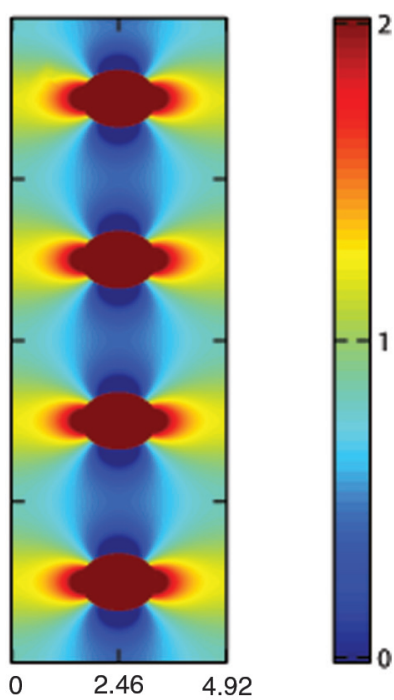

d)

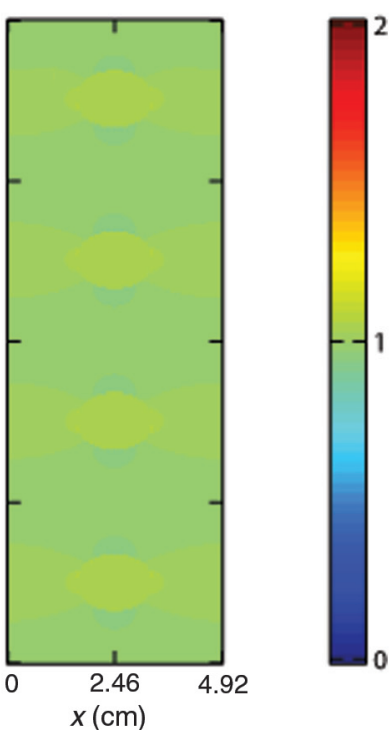

Figure 11. Normalized vertical (a and c) stress and (b and d) strain fields inferred from creep tests for ( $a$ and $b$ ) a typical sand and ( $c$ and d) sandstone in the high-frequency limit for the case of patchy saturation. case, the resulting vertical stress field is homogeneous throughout the entire sample with the very exception of tiny regions at the fracture tips. As a consequence, estimates of the effective P-wave modulus inferred from creep and relaxation tests are equivalent for RUCs of any size because no boundary condition effects occur. This result is consistent with what we observed in the considered case of patchy saturation. Also for this scenario, we verified that the effective $\mathrm{P}$-wave modulus obtained with the numerical tests in the

a)

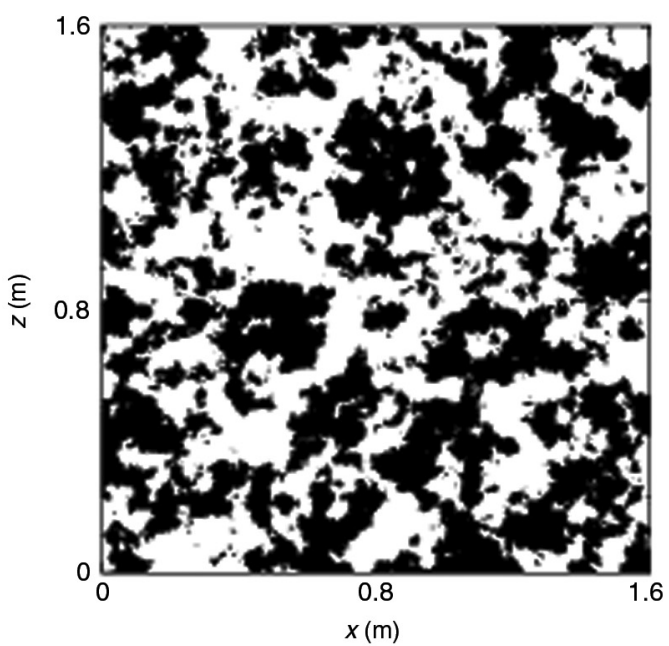

b)

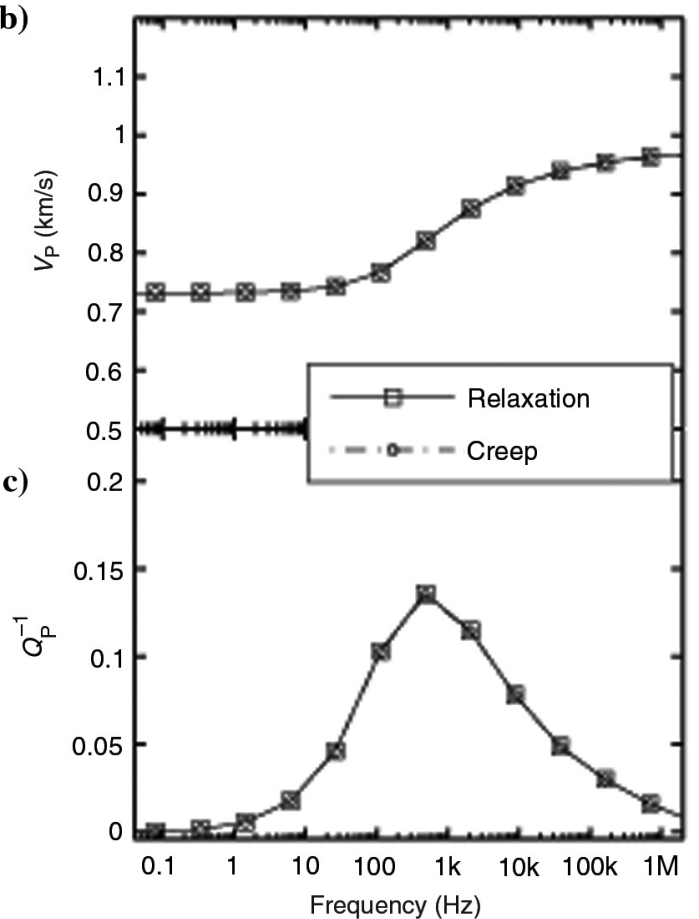

Figure 12. (a) Strongly heterogeneous, fractal-type patchy saturation distribution of gas (black regions) and water (white regions) obtained from the corresponding stochastic von-Kármán-type distributions by thresholding. The overall gas saturation corresponds to $S_{g}=50 \%$. The physical properties of the grain, matrix, and the saturating pore fluids are given in Table 2. (b) P-wave phase velocity and (c) inverse quality factor inferred from relaxation (squares) and creep (circles) tests. 
high-frequency limit is properly consistent with the known averaging rule $H^{-1}=\langle 1 / H\rangle$ (Hill, 1963).

Another attenuation mechanism, which is mainly driven by stiffness contrasts, is squirt flow. This mechanism is due to fluid flow at the microscopic scale between cracks and pores and between connected cracks, where cracks and pores have a similar spatial scale (Adelinet et al., 2011; Guéguen and Sarout, 2011). In the presence of connected mesoscopic fractures, WIFF within the fractures is conceptually very similar to squirt flow at the microscopic scale (Rubino et al., 2013a, 2014; Quintal et al., 2015). Although these mechanisms operate at fundamentally different scales, the insights gained in this study are therefore expected to be transferrable to the estimation of REV sizes in the presence of squirt flow.

The fundamental question regarding the definition of REVs explored in this work will naturally persist for more complex poroelastic media, such as realistic fracture networks. For these random media, we neither expect a periodic distribution of the stress and strain fields nor a constant stress field at the boundaries. Correspondingly, the stress and strain fields are likely to be disturbed at the boundaries for relaxation and creep tests. Furthermore, the overall statistical properties of the underlying heterogeneous medium have to be captured by the considered subvolumes to fulfill Hill's (1963) first condition of an REV. This can be achieved by estimating the mean and variance of the upscaled $\mathrm{P}$-wave velocity and attenuation from simulations over many realizations on a given subvolume of the random medium (Caspari et al., 2015). However, the obtained mean values will be biased by boundary condition effects if the size of the subvolume does not fulfill Hill's (1963) second condition. Therefore, the results of this study provide valuable insights on how fracture density, stiffness contrast, and the presence of patchy saturation influence these boundary condition effects. In fact, a periodic arrangement of fractures, despite its simplicity, is the most severe case with regard to boundary condition effects, and thus the obtained results are expected to hold for more general scenarios of random media. For random fracture distributions, the competing effects of the shielding and amplification zones may cancel out, thus resulting in more homogeneous stress fields (Kachanov, 1992).

The computational upscaling procedures considered in this study also have the potential to assist the interpretation of experimentally determined phase velocity and attenuation estimates and the design of laboratory measurements. In a laboratory setting using the forced oscillation method, typical sample sizes are of the order of few centimeters in the diameter and length and the estimates of attenuation are based on the application of homogeneous stress fields and measurements of resulting strains (Subramaniyan et al., 2014). In such samples, mesoscopic heterogeneities in the form of fluid patches are thought to exist in the millimeter to centimeter range. Given that, as indicated by the results of this study, boundary condition effects appear to be negligible for this scenario in the absence of strong mesoscopic heterogeneity of the solid matrix, the interpretation of corresponding experimental findings may thus indeed be applicable beyond the laboratory scale. The same applies for horizontally layered samples as long as Hill's (1963) first condition regarding statistical stationarity is satisfied. Conversely, if the sample contains mesoscopic fractures, as sometimes evidenced by X-ray tomographic scans, our results indicate that, depending on the degree of interaction between the fractures and the prevailing boundary conditions, the observed velocity dispersion and attenuation characteristics may not be representative of larger rock masses.

\section{CONCLUSIONS}

We have analyzed the effects of different boundary conditions on the estimation of P-wave phase velocity and attenuation in periodically heterogeneous poroelastic media. We verified that an adequate estimation of REVs is possible through a convergence analysis of the seismic properties inferred from relaxation and creep tests applied to RUCs composed of an increasing number of fundamental blocks. It is important to note that relaxation and creep tests correspond to two limiting sets of boundary conditions. A systematic analysis performed on a range of periodically heterogeneous rock samples associated with high attenuation allowed us to infer some potential implications for practical applications.

Our numerical results indicate that boundary condition effects, and therefore the sizes of the REVs, are mainly controlled by the degree of interaction between the stress and strain fields of adjacent heterogeneities, which in turn depend on the separation between the heterogeneities, the stiffness contrasts of the heterogeneous solid matrix, and the frequency. In particular, we observe that boundary condition effects do not occur in periodically layered poroelastic media, which is a direct consequence of the stress and strain distribution within the sample related to the dimensionality of the system. Furthermore, boundary condition effects tend to be negligible for porous media characterized by patchy saturation in spite of the potentially very high compressibility contrasts between the different saturating pore fluids. Conversely, strong boundary condition effects prevail in fractured media for a realistic range of stiffness contrasts. Furthermore, the observed frequency dependence of the boundary condition effects, which causes an overestimation of velocity dispersion and attenuation for creep tests, is a characteristic feature of poroelastic systems that has no equivalent for heterogeneous elastic media. The results of this study, therefore, point to the importance of carefully determining the sizes of REVs whenever effective seismic properties of heterogeneous porous media are considered, particularly so in the presence of strong dry frame stiffness contrasts.

\section{ACKNOWLEDGMENTS}

This work has been supported by a grant from the Swiss National Science Foundation. T. M. Müller gratefully acknowledges an extended visit to the University of Lausanne financed by the Fondation Herbette. We wish to thank Y. Guéguen, E. Saenger, and an anonymous referee for their insightful comments and suggestions, which greatly helped to improve the quality of this paper.

\section{APPENDIX A}

\section{BOUNDARY CONDITIONS FOR THE NUMERICAL RELAXATION AND CREEP TESTS}

To estimate the equivalent P-wave modulus (equation 14) of a porous sample subjected to an oscillatory relaxation or creep test, we solve the Biot's (1941) quasi-static poroelastic equations 1-4 using the following boundary conditions. Let us define the computational domain area $\Omega=L_{x} \times L_{z}$ whose boundary $\Gamma$ is given by $\Gamma=\Gamma^{L} \cup \Gamma^{B} \cup \Gamma^{R} \cup \Gamma^{T}$, where

$$
\Gamma^{L}=\{(x, z) \in \Omega: x=0\},
$$




$$
\begin{gathered}
\Gamma^{R}=\left\{(x, z) \in \Omega: x=L_{x}\right\}, \\
\Gamma^{B}=\{(x, z) \in \Omega: z=0\}, \\
\Gamma^{T}=\left\{(x, z) \in \Omega: z=L_{z}\right\} .
\end{gathered}
$$

Here, $\nu$ and $\chi$ denote the unit outer normal and unit tangent on $\Gamma$ so that $\{\boldsymbol{\nu}, \boldsymbol{\chi}\}$ is an orthonormal system on $\Gamma$. In the case of relaxation tests, the considered set of boundary conditions is

$$
\begin{array}{r}
\boldsymbol{u} \cdot \boldsymbol{v}=-u^{0}, \quad(x, z) \in \Gamma^{T}, \\
\boldsymbol{u} \cdot \boldsymbol{v}=0, \quad(x, z) \in \Gamma^{L} \cup \Gamma^{R} \cup \Gamma^{B}, \\
\boldsymbol{w} \cdot \boldsymbol{v}=0, \quad(x, z) \in \Gamma, \\
(\boldsymbol{\sigma} \boldsymbol{\nu}) \cdot \chi=0, \quad(x, z) \in \Gamma,
\end{array}
$$

and for creep tests we have

$$
\begin{gathered}
\boldsymbol{\sigma} \boldsymbol{\nu}=\left(0,-\sigma^{0}\right), \quad(x, z) \in \Gamma^{T}, \\
(\boldsymbol{\sigma} \boldsymbol{\nu}) \cdot \chi=0, \quad(x, z) \in \Gamma^{L} \cup \Gamma^{R} \cup \Gamma^{B}, \\
\boldsymbol{u} \cdot \boldsymbol{v}=0, \quad(x, z) \in \Gamma^{L} \cup \Gamma^{R} \cup \Gamma^{B}, \\
\boldsymbol{w} \cdot \boldsymbol{v}=0, \quad(x, z) \in \Gamma .
\end{gathered}
$$

Fixing the bottom boundary imposes a mirror symmetry on the considered sample. This means that the effective P-wave modulus resulting from these boundary conditions is equivalent to what we would obtain by prescribing either a stress or a displacement to the top and the bottom boundaries of a sample that has twice the size with the lower half corresponding to a reflected image of the sample considered when fixing the bottom boundary (Drago and Pindera, 2007).

\section{APPENDIX B}

\section{BOUNDARY CONDITION EFFECTS FOR A STO- CHASTIC DISTRIBUTION OF FLUID PATCHES}

In this appendix, we verify whether our finding regarding the generic absence of boundary condition effects in the presence of patchy saturation also applies for a highly heterogeneous stochastic distribution of fluid patches. To this end, we compare the P-wave phase velocity and inverse quality factor inferred from relaxation and creep tests for the rock sample shown in Figure 12a. This band-limited fractal distribution of the gas and fluid patches is obtained from a stochastic von Kármán-type distribution through thresholding (Tronicke and Holliger, 2005). The overall gas saturation is $S_{g}=50 \%$. We use the grain and matrix properties corresponding to those of an unconsolidated clean sand (Table 2), which, in terms of the potential boundary condition effects, is expected to correspond to the worst-case scenario. The equivalence of the Pwave phase velocity and inverse quality factor inferred from relaxation and creep tests (Figure 12b and 12c) corroborates our generic finding that no boundary condition effects occur in the presence of patchy saturation.

\section{REFERENCES}

Adelinet, M., J. Fortin, and Y. Guéguen, 2011, Dispersion of elastic moduli in a porous-cracked rock: Theoretical predictions for squirt-flow: Tectonophysics, 503, 173-181, doi: 10.1016/j.tecto.2010.10.012.

Biot, M., 1941, General theory of three-dimensional consolidation: Journal of Applied Physics, 12, 155-164, doi: 10.1063/1.1712886.

Biot, M., 1956a, Theory of propagation of elastic waves in a fluid-saturated porous solid. I. Low-frequency range: Journal of the Acoustical Society of America, 28, 168-178, doi: 10.1121/1.1908239.

Biot, M., 1956b, Theory of propagation of elastic waves in a fluid-saturated porous solid. II. Higher frequency range: Journal of the Acoustical Society of America, 28, 179-191, doi: 10.1121/1.1908241.

Caspari, E., M. Milani, J. G. Rubino, T. M. Müller, B. Quintal, and K. Holliger, 2015, Numerical upscaling of seismic characteristics of strongly heterogeneous poroelastic media: Randomly distributed parallel fractures: Presented at the 3rd International Workshop on Rock Physics Technical Program.

Drago, A. S., and M.-J. Pindera, 2007, Micro-macromechanical analysis of heterogeneous materials: Macroscopically homogeneous vs periodic microstructures: Composites Science and Technology, 67, 1243-1263, doi: 10.1016/j.compscitech.2006.02.031

Gitman, I. M., H. Askes, and L. J. Sluys, 2007, Representative volume: Existence and size determination: Engineering Fracture Mechanics, 74 2518-2534, doi: 10.1016/j.engfracmech.2006.12.021.

Grechka, V., and M. Kachanov, 2006, Effective elasticity of fractured rocks: A snapshot of the work in progress: Geophysics, 71, no. 6, W45-W58, doi: 10.1190/1.2360212.

Guéguen, Y., and J. Sarout, 2011, Characteristics of anisotropy and dispersion in cracked medium: Tectonophysics, 503, 165-172, doi: 10 .1016/j.tecto.2010.09.021.

Hill, R., 1963, Elastic properties of reinforced solids: Some theoretical principles: Journal of the Mechanics and Physics of Solids, 11, 357-372, doi: 10.1016/0022-5096(63)90036-X.

Huet, C., 1990, Application of variational concepts to size effects in elastic heterogeneous body: Journal of the Mechanics and Physics of Solids, 38, 813-841, doi: 10.1016/0022-5096(90)90041-2.

Huet, C., 1999, Coupled size and boundary-condition effects in viscoelastic heterogeneous and composite bodies: Mechanics of Materials, 31, 787829, doi: 10.1016/S0167-6636(99)00038-1.

Jaeger, J. C., N. G. W. Cook, and R. W. Zimmerman, 2007, Fundamentals of rock mechanics, 4th ed.: Blackwell Publishing.

Jänicke, R., B. Quintal, and H. Steeb, 2015, Numerical homogenization of mesoscopic loss in poroelastic media: European Journal of Mechanics A/ Solids, 49, 382-395, doi: 10.1016/j.euromechsol.2014.08.011.

Johnson, D. L., 2001, Theory of frequency dependent acoustics in patchysaturated porous media: Journal of the Acoustical Society of America, 110, 682-694, doi: 10.1121/1.1381021.

Kachanov, M., 1992, Effective elastic properties of cracked solids: critical review of some basic concepts: Applied Mechanics Reviews, 45, 304335, doi: 10.1115/1.3119761.

Knott, J. F., 1973, Fundamentals of fracture mechanics: Butterworths.

Kundu, T., 2008, Fundamentals of fracture mechanics: CRC Press.

Masson, Y. J., and S. R. Pride, 2007, Poroelastic finite difference modeling of seismic attenuation and dispersion due to mesoscopic-scale heterogeneity: Journal of Geophysical Research, 112, B03204, doi: 10.1029/ 2006JB004592.

Mavko, G., T. Mukerji, and J. Dvorkin, 2009, The rock physics handbook: Tools for seismic analysis of porous media: Cambridge University Press.

Müller, T. M., B. Gurevich, and M. Lebedev, 2010, Seismic wave attenuation and dispersion resulting from wave-induced flow in porous rocks A review: Geophysics, 75, no. 5, 75A147-75A164.

Ostoja-Starzewski, M., 2006, Material spatial randomness: From statistical to representative volume element: Probabilistic Engineering Mechanics, 21, 112-132, doi: 10.1016/j.probengmech.2005.07.007.

Pride, S. R., 2005, Relationships between seismic and hydrological properties, in Y. Rubin, and S. Hubbard, eds., Hydrogeophysics: Springer, 253-291.

Pride, S. R., J. G. Berryman, and J. M. Harris, 2004, Seismic attenuation due to wave-induced flow: Journal of Geophysical Research, 109, B01201.

Quintal, B., J. G. Rubino, E. Caspari, and K. Holliger, 2015, A simple hydromechanical approach to simulate squirt-type flow at any scale: A simple hydro-mechanical approach to simulate squirt-type flow at any scale: Annual International Meeting, SEG, Expanded Abstracts, 3224-3228.

Rubino, J. G., L. Guarracino, T. M. Müller, and K. Holliger, 2013a, Do seismic waves sense fracture connectivity?: Geophysical Research Letters, 40, 692-696, doi: 10.1002/grl.50127.

Rubino, J. G., L. Guarracino, T. M. Müller, and K. Holliger, 2013b, Seismic wave attenuation and dispersion due to wave-induced fluid flow in rocks 
with strong permeability fluctuations: Journal of the Acoustical Society of America, 134, 4742-4751, doi: 10.1121/1.4824967.

Rubino, J. G., T. M. Müller, L. Guarracino, M. Milani, and K. Holliger, 2014, Seismoacoustic signatures of fracture connectivity: Journal of Geophysical Research, 119, 2252-2271.

Rubino, J. G., C. L. Ravazzoli, and J. E. Santos, 2009, Equivalent viscoelastic solids for heterogeneous fluid-saturated porous rocks: Geophysics, $\mathbf{7 4}$ no. 1, N1-N13, doi: 10.1190/1.3008544.

Schön, J. H., 2004, Physical properties of rocks: Fundamentals and principles of petrophysics: Elsevier.

Solazzi, S. G., J. G. Rubino, T. M. Müller, M. Milani, L. Guarracino, and K. Holliger, 2014, An energy-based approach to estimate seismic attenuation due to wave-induced fluid flow: 84th Annual International Meeting, SEG, Expanded Abstracts, 1991-1995.

Subramaniyan, S., B. Quintal, N. Tisato, E. H. Saenger, and C. Madonna, 2014, An overview of laboratory apparatuses to measure seismic attenuation in reservoir rocks: Geophysical Prospecting, 62, 1211-1223, doi: 10 $.1111 /$ gpr.2014.62.issue-6.

Tronicke, J., and K. Holliger, 2005, Quantitative integration of hydrogeophysical data: Conditional geostatistical simulation for characterizing heterogeneous alluvial aquifers: Geophysics, 70, no. 3, H1-H10, doi: $10.1190 / 1.1925744$

White, J. E., 1975, Computed seismic speeds and attenuation in rocks with partial gas saturation: Geophysics, 40, 224-232, doi: 10.1190/1.1440520. 